\title{
Successful Management of Peripartum Cardiomyopathy
}

\author{
*SF Siddiqua ${ }^{1}$, S Abbasi² ${ }^{2}$ H Haque ${ }^{3}$, AS Hasan ${ }^{4}$ \\ $1 *$ Prof. Dr. Sehereen F.Siddiqua, Head, Dept of Gynae and obst, Anwer khan Modern Medical College Hospital \\ ${ }^{2}$ Dr. Sharmin Abbasi, Asst. Professor, Dept of Gynae and Obst Anwer khan Modern Medical College Hospital \\ ${ }^{3}$ Dr. Harisul Haque, Assoc. Prof. Dept of Cardiology, Banghabandhu Sheikh Mujib medical University \\ ${ }^{4}$ Dr. Abrar Saqif Hasan, Intern doctor, Ibrahim Medical College and BIRDEM \\ *Corresponding Author
}

Date of submission: 22.11.2015 Date of acceptance: 04.06.2016

\begin{abstract}
Peripartum cardiomyopathy is a rare form of cardiac disorder of unknown origin associated with a high risk of mortality $(50 \%)$. Echocardiogram confirms the diagnosis by typically reveals a left ventricular dialatation and a decreased ejection fraction (45\%). Up to date, the treatment remains symptomatic. The prognosis is variable, ranging from complete recovery to worsening heart failure. Future pregnancy often discourge because of high mortality rate and risk of recurrence. Prognosis is related to recovery of ventricular fuction. Among many cases we report 3 more complicated cases of peripartum cardiomyopathy that managed successfully in our hospital.
\end{abstract}

Key Words: Peripartum, cardiomyopathy, pregnancy, heart failure, left ventricular dysfunction, ejection fraction.

\section{Introduction}

Peripartum cardiomyopathy (PPCM) is an idiopathic cardiomyopathy, characterized by heart failure, left ventricular systolic dysfunction towards the end of pregnancy or within 5 months of delivery, when no other causes of heart failure is found ${ }^{2}$.

The exact pathogenetic mechanism is not fully understood. Several pathogenetic factors may be associated: inflammatory, infectious, genetic , autoimmune, oxidative stress. The diagnosis is confirmed on the basis of diagnostic criteria : a) Development of heart failure during the last month of pregnancy or within in 5 months of delivery, b) Absence of identifiable causes for heart failure c) Absence of recognizable heart disease prior to the last month of pregnancy. d) Left ventricular dysfunction determined during echocardiography with ejection fraction $<45 \%$. PPCM treatment is not different from acute and chronic heart failure treatment. Discontinuation of therapy is recommended only in case of recovery of the left ventricular function, gradually monitoring cardiac function and repeating the 2D cardiac ultrasound imaging. The recovery of the left ventricular function usually occurs within $2-6$ months of diagnosis. The

AKMMC J 2017; 8(1): 67-70 mortality rate ranges from $1 \%$ to $19 \%$. Repeated risk of PPCM during subsequent pregnancy is 30$50 \%$ if the ventricular dysfunction remains 3 .

\section{Case History}

Case:1

Our case is about a 33 years old women, from middle class family, para $2(1 \mathrm{NVD}+1 \mathrm{C} / \mathrm{S})$. The first one was delivered vaginally at home without any known complications and second one was delivered in by elective $\mathrm{C} / \mathrm{S}$ due to preeclampsia with fetal distress in outer hospital. In fact, the recent pregnancy was poorly monitored. It was $7^{\text {th }}$ day of puerperium, the patient was admitted to OBGYN emergency of AKMMCH with sever respiratory distress NYHA stage 3 and also associated to orthopnea, sever cough and frothy sputum. The overall examination showed - patient was obese, conscious with profuse sweating, heart rate was $112 \mathrm{bpm}$, respiratory rate was 34 cycles per min with high BP 180/110 mm of $\mathrm{Hg}$, edema present. The patient reported a quick weight gain since a month. Proteinuria was positive $(3+)$ which it was in favor of severe pre eclampsia. The chest 
auscultation showed crackles at its base. The obstretical examination revealed fundal height 12 $\mathrm{cm}$, uterus was firm in consistency and non tender. Lochial discharge was normal which indicates proper involution. The electrocardiogram was in favor of an acute pulmonary edema. Echocardiography showed a dilated left ventricle, global hypokinesia and left ventricular hypertension. Left ventricular ejection fraction was $42 \%$. The left atrium was dilated, the right cavities were non dilated and the right ventricle systolic function was normal. These results showed the impresson of dialated cardiomyopathy.chest x-ray showed pneumonic consolidation The patient was quickly resuscitated (half sitting position, oxygen, urinary catheterization). A biological assessment with: $\mathrm{CBC}$, Serum electrolyte, with liver function and renal function, CRP, lipid profile, thyroid function, cardiac enzymes are made. The blood count was unremarkable, the electrolytes, the liver and renal function tests were normal, CRP was elevated at $17.4 \mathrm{mg} / \mathrm{ml}$. The thyroid function test are normal and troponin $\mathrm{I}$ and $\mathrm{C}$ was negative. Diuretics therapy was started. The blood pressure was high and calcium channel inhibitor and labetelol was continued. Prophylactic heparin also started and continued up to 5 days. An inhibitor of lactation was givan to prevent breast engorgement. During the hospital course blood pressure returned to normal in the twelve day of the post-partum period and Clinically overall improvement was observed. The last echocardiography before discharge found an ejection fraction of $52 \%$ which was increased than before. After six days of hospitalization and a good clinical course, the patient was discharged to home.

\section{Case:2}

A 36 years-old lady came to OBGYN OPD at 37 weeks of pregnancy with the complaints of sudden attack of respiratory distress. It was her second pregnancy. She had no known heart disesase and she was normotensive and non diabetic. She was in regular antenatal cheakup. Up to 37 weeks her pregnancy was uneventful. She was a known case of hypothyroidism and she was euthyroid in whole pregnancy by thyroxin replacement therapy. On admission pt had orthopnea and cough. Examination showed heart rate $110-120$ beats/min, RR 32/min. BP:110/70 $\mathrm{mm}$ of $\mathrm{Hg}$. The obstetrical examination revealed fundal height: $35 \mathrm{~cm}$ with cephalic
SF Siddiqua, S Abbasi, H Haque, AS Hasan et al

presentation. Fetal heart sound was well perceived .Ultrasound of pregnancy profile showed-cephalic presentation with fetal weight $3.2 \mathrm{~kg}$, placenta anterior, AFI:14 cm. BPP 6/10.Umbilical and cerebral doppler were normal. The echocardiogram showed : left ventricular dysfuction with global hypokinesia. LVEF: 32\%.Chest X-ray with abdominal protector was in favour of an acute pulmonary edema. On the Doppler, there was aitral regurgitation grade 1-1l and a tricuspid insufficiency grade 1 with significant pulmonary hypertension. The pericardium was dry. These results was in favour of dilated cardiomyopathy. Troponin I was also raised. The patient was quickly managed by -propped up position. High flow oxygen given and urinary catheterization. She received loop diuretics $40 \mathrm{mg}$ $\mathrm{I} / \mathrm{V}$ stat and others symptomatic management.Others Parameters in blood was normal. A medical board was held by the presence of cardiologist, ICU specialist, anesthesiologist and medicine internist. The fate of the patient was well explained to party and they agreed for $\mathrm{c} / \mathrm{s}$ with the high risk bond. An emergency $\mathrm{C} / \mathrm{S}$ was carried out under $\mathrm{G} / \mathrm{A}$ and a healthy male baby with $3 \mathrm{~kg}$ was delivered. After $\mathrm{C} / \mathrm{S}$, the patien was transferred to the ICU. Diuretic therapy was continued. Prophylactic heparin therapy was given to prevent thromboembolism. After five days in ICU the patient transferred to CCU.After 7 days patient transferred from $\mathrm{CCU}$ to Obs ward. Clinically patient was improved after14 days and discharged to home. After three weeks Echocardiography result was improved-EF was $55 \%$.

\section{Case:3}

A 32 years old para $(2 \mathrm{C} / \mathrm{S}+0)$ came to gynae and obst emergency 1 week after LUCS with sever respiratory distress. Pt state that she had dyspnea during lying position but it improves by sitting. On examination pt was drowsy, mild pallor present with respiratory distress.Her BP was $90 / 60 \mathrm{~mm}$ of $\mathrm{Hg}$, heart rate was 124 beats $/ \mathrm{min}$. RR was $33 / \mathrm{min}$. She was afebrile. Chest examination showed-bilateral basal crepitation. Cardiovascular and abdominal examination was unremarkable. Initial laboratory investigation were normal except gross cardiomegaly in Chest $\mathrm{x}$-ray and Echocardiogram showed EF-28\%. After initial resuscitation pt treated by loop diuretics I/V 12 hourly . Her condition was not improving. So the pt transferred to $\mathrm{CCU}$ for further proper management and digitilization was done. But the recovery was very 
slow. After 2 weeks in CCU stay pt was transferred to cabin. She was switched over to oral digoxin therapy. After 7 days repeat echocardiogram report showed-EF-31\%.So it was suggestive of very slow improvement. She was advised to continue regular medical follow up. Her echocardiography during follow up after 2 months showed normal left ventricular size and contractility with normal ejection fraction.
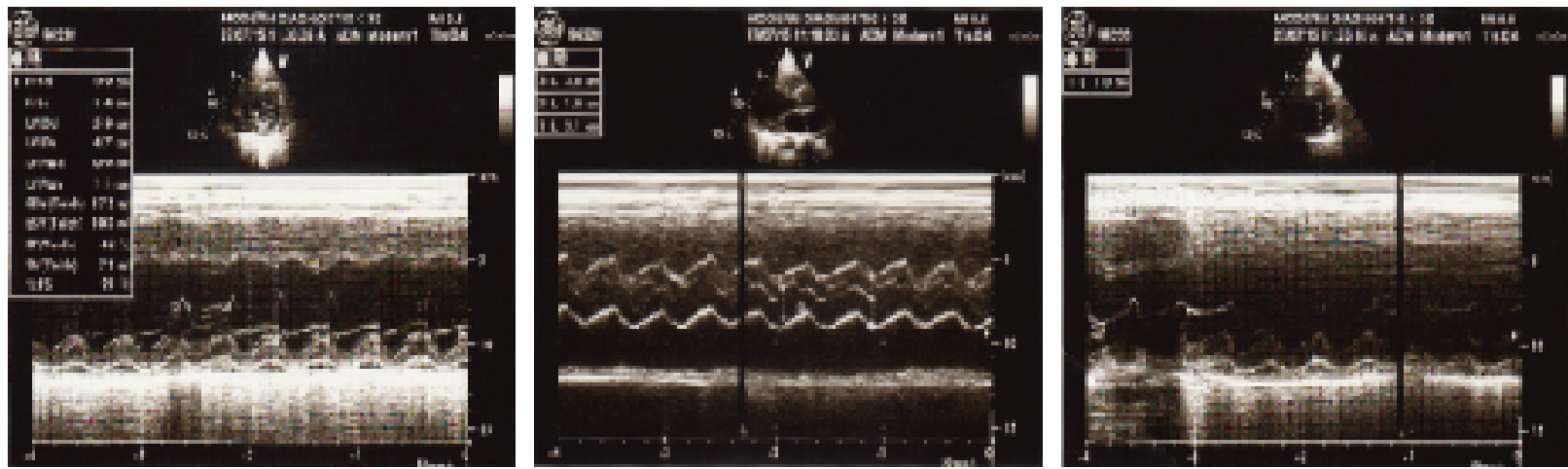

\section{Discussion}

Peripartum cardiomyopathy has been defined by the working group on Heart failure of the European Society of Cardiology as an idiopathic cardiomyopathy manifested by signs of heart failure secondary to left systolic dysfunction between the last month of pregnancy and the five months following delivery when no other etiology was found. Dilatation of the left ventricle is not compulsory but the left ventricle ejection fraction is always less than $45 \%$. Several risk factors for PPCM were identified: Maternal age $>30$ years, multiparity ,multiple pregnancy, obesity, high blood pressure, pre-eclampsia. In fact, in $1 / 3$ of cases, no risk factor was found. Our patients had four of these risk factors. The clinical picture of PPCM is systolic heart failure. Functional signs are dyspnea, cough, edema of the lower limbs. The cardiopulmonary auscultation found crackles or wheezes related to pulmonary congestion. Our patients presented with the same clinical picture. The differential diagnosis includes pulmonary embolism, pneumonia, infarction and aortic dissection. The ECG usually shows sinus tachycardia but atrial arrhythmias are not uncommon. Besides, left ventricular hypertrophy, ischemia in epicardial Q waves, ST segment changes may also present. InECG our patients showed sinus tachycardia at more than 100 beats/min with some atrial extra systoles. CXR is not specific for showing signs of heart failure and inconstant cardiomegaly. Successive radiography will allow to follow the evolution of congestive signs. In our cases, CXR objectified an acute pulmonary edema. Echocardiography is the key examination for positive and differential diagnosis, prognostication, therapeutic decision making and follow up. She often found a left ventricular dilatation, decreased less than $45 \%$ ejection fraction and possible left ventricular disease associated. Finally, it is often used to confirm the diagnosis of PPCM by eliminating pre-existing heart disease (hypertrophic, rheumatic valvular, ischemic). It assesses the severity of cardiac diseases and follow the evolution: Troponin I or $\mathrm{T}$ related to myocyte necrosis, C-reactive protein reflecting the scalability of inflammatory processes, transaminases, creatinine and lactate, to asess the visceral impact of heart failure. The evolution of the PPCM is often extremely fast and unpredictable with possibility of sudden onset of refractory cardiogenic shock in the first 24-48 hours. The treatment of PPCM in acute phase is symptomatic.Diuretics is used to reduce preload and afterload and increases myocardial contractility. Several drugs are useable depending on the clinical features and the period of pregnancy. Decreasing the precharge can be provided by nitro derivatives and diuretics. ACEI are the best treatment to decrease afterload but they are contraindicated in pregnancy due to side effects of the foetus (prematurity, oligoamnios, bone malformation $)^{4}$. Amlodipine or hydralazine-nitro derivatives association are alternatives during pregnancy. Beta-blockers are prescribed during tachycardis or arrhythmis. Dobutamine increases myocardial contractility without risk during pregnancy. Therapeutic abortion sometimes be justified in case of worsening heart failure despite optimal medical therapy. The duration of this long term medical treatment is not well defined. It must 
be at least one year and then based on ultrasound monitoring. Some authors advocate unconventional treatments as immunosuppressive. Plasmapheresis, immunoglobulin, interferon or immunomodulations for patients who remain symptomatic under conventional medical treatment and in whom myocarditis is proved by endomyocardial biopsy. A small randomized study published in 2010 has opened up new perspective. This open study comparing 20 patients placed under bromocriptine versus conventional treatment isolated, showed a benefit effects in terms of improvement in LVEF to 4 months. Other complaications associated to PPCM include thromboembolic events which are found in nearly $50 \%$ of cases. The hypercoagulability secondary to pregnancy and blood stasis in dilated cervicitiis promote the formation of thrombi. In fact, anticoagulation is justified and should be pursed as ejection fraction is less than $35 \%$. The management of the patients with PPCM is multidisciplinary including obstetrician, cardiologist, anesthetist and pediatrician. Child birth can be done vaginaly and epidural analgesia is the medically indicated, as it reduces the myocardial work during labour and improvement in cardiac function by decreasing load conditions. An indication for $\mathrm{C} / \mathrm{S}$ may be raised by the obstetrician or in case of severe cardiac decompression. General or regional anesthesia in then used, The goal is to maintain a good hemodynamic stability. Spinal anesthesia alone is strongly discouraged because of the sudden hemodynamic changes it engenders and which can be fatal in this context. Epidural anesthesia and combined spinal anesthesia are preferred. The regional anesthesia is preferred except in case of emergency obstetric and contraindicated, If the PPCM occurs in the last months of pregnancy, in order to benefit the mother of a better therapeutic management, rapid extraction of the foetus is often indicated. If the cervix is ready and If maternal hemodynamic status permits, vaginal delivery may be authorized. Recurrence of PPCM during a subsequent pregnancy occurs in 25$100 \%$ of cases depending on the study. Finally, European society of cardiology recommends to ${ }^{5}$

$\Rightarrow$ Discourage a future pregnancy in a patient with ventricular sequelae post PPCM.

$\Rightarrow$ Against state of pregnancy if LVEF $<50 \%$

$\Rightarrow$ Perform a therapeutic abortion in case of deterioration of $\mathrm{LVEF}<50 \%$ in the 1 st or $2^{\text {nd }}$
SF Siddiqua, S Abbasi, H Haque, AS Hasan et al

quarter, due to the risk of heart failure may jeopardize the mother's prognosis.

$\Rightarrow$ Perform echocardiography screening and regular monitoring in patients with history of PPCM with normalization of LVEF, as well as in patients with a family history of cardiomyopathy or PPCM ascendants and collaterals in the first degree. ${ }^{6}$

\section{Conclusion}

Due to its rarity, variability in presentation, and potential for mortality, PPCM should be considered in women that present with features consistent with left ventricular failure. A Secondary normalization of ventricular function does not exclude a risk of recurrence in subsequent pregnancy justifying a multidisciplinary long term monitoring. Though it should be borne in mind that this can mimic the normal physiological changes of pregnancy.

Conflict of interest: Authors declared that they have no conflict of interest.

\section{References}

1. H. Wilkinson," Saving Mothers lives. Reviewing maternal deaths to make motherhood safer: 2006-2008, International journal of Obstetrics and Gynaecology" 2011; 118(11): 1402-1403.

2. K. Sliwa, D. Hilfiker-Kleiner, M.C. Petrie et al., "Current state of knowledge an aetiology, diagnosis, management, and therapy of peripartum cardiomyopathy: a position statement from the Heart Failure Association of the European Society of Cardiology working group on peripartum cardipmyopathy", European journal of heart failure, 2000; 12(8):767-778.

3. E. P. Gunderson, L. A. Croen, V. Chiang, "Epidemiology of peripartum cardiomyopathy: incidence, predicators, and outcomes, "Obstetrics and Gyenocology, 2000; 118(3): 583-591.

4. R. Ramaraj and V. L. Sorrell, "Peripartum cardiomyopathy causes, diagnosis and treatment," Cleveland clinical journal of medicine, 2011; 76(5): 289-296.

5. J.D. Fett and A.A. Ansari, "Inflammatory markers and cytokines in peripartum cardiomyopathy : a delicate balance,"Expert Opinion on therapeutic targets, 2011; 14(9): 895-898.

6. S. Lamparter, S. Pankuweit and B. Maisch, "clinical and immunologic charasterstics in peripartum cardiomyopathy, "International journal of cardiology, 2007; 118(1): 14-20. 